\title{
APPLIED VISUAL EXPLORATION ON REAL-TIME NEWS FEEDS USING POLARITY AND GEO-SPATIAL ANALYSIS
}

\author{
Miloš Krstajić, Peter Bak, Daniela Oelke, Daniel A. Keim \\ Department of Computer and Information Science, University of Konstanz, Germany \\ \{krstajic, bak, oelke, keim\}@dbvis.inf.uni-konstanz.de \\ Martin Atkinson \\ European Commission's Joint Research Center, Ispra, Italy \\ martin.atkinson@jrc.it \\ William Ribarsky \\ UNC Charlotte Visualization Center, Charlotte, U.S.A. \\ ribarsky@uncc.edu
}

Keywords: News feed application, Sentiment analysis, Spatiotemporal analysis.

\begin{abstract}
This paper presents a visual analytics approach to explore large news article collections in the domains of polarity and spatial analysis. The exploration is performed on the data collected with Europe Media Monitor (EMM), a system which monitors over 2500 online sources and processes 90,000 articles per day. By analyzing the news feeds, we want to find out which topics are important in different countries and what is the general polarity of the articles within these topics. To assess the polarity of a news article, automatic techniques for polarity analysis are employed and the results are represented using Literature Fingerprinting for visualization. In the spatial description of the news feeds, every article can be represented by two geographic attributes, the news origin and the location of the event itself. In order to assess these spatial properties of news articles, we conducted our geo-analysis, which is able to cope with the size and spatial distribution of the data. Within this application framework, we show opportunities how real-time news feed data can be analyzed efficiently.
\end{abstract}

\section{INTRODUCTION}

Excess amount of information is generated each day on the internet, making processing of the content very difficult for the individual. Global news agencies, such as The Associated Press (AP), Reuters and Agence France-Presse (AFP), provide media companies with news reports from all over the world. This content is then duplicated, enriched with commentary and opinion. Additionally, news are filtered according to importance or interest of the editorial team. Besides, local media outlets produce their own local (or global) content having their own point of view, which might be specific to the geographic location of the news source (region, country) or specific to a certain group of people. Furthermore, blogs allow common people to become active content creators themselves, not just passive readers, thus making the analysis of such amount of information one of today's greatest challenges.

The current paper describes an application aiming to conduct comprehensive analysis of such material. The paper first describes where the data comes from and how it is processed for analytic purposes. Second, opportunities for in-depth analysis are shown, taking polarity and spatial analytic techniques as examples.

Europe Media Monitor (Atkinson and Van der Goot, 2009) is a news aggregation system which monitors over 2500 news sources, collecting 80,000 100,000 news articles per day in 42 languages. The goal of EMM is to provide assistance to human media monitoring, through automatic analysis and categorization of articles from these sources. In a typical information gathering scenario, journalists try to give the answers to the "Five Ws" questions - "who, what, when, where and why". The EMM system employs various information extraction, clustering and analysis techniques to help the user in answering these questions. The front-end application EMM NewsBrief is available publicly at http://emm.jrc.it/.

Articles are clustered by the EMM system in each 
language into stories that report about the same event. Each article is enriched with various metadata, such as people, their titles and organizations which are mentioned in the articles. This data is extracted in a separate entity recognition process and is available in all languages. Figure 1 shows an example with German Prime Minister Angela Merkel. In order to give the answer to the question "where?" about the story location, geographical information is also extracted. The disambiguation module in the system uses the meta-information of previously recognized entities, such as names of places, provinces, regions and countries, in order to perform geo-tagging.
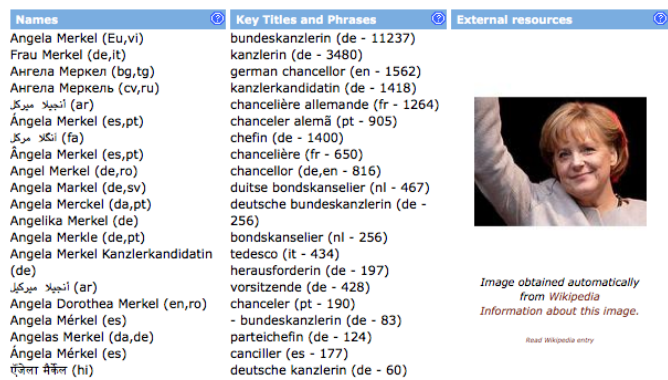

Figure 1: EMM Website Person Page provides data about people, their titles and quotations in multiple languages.

Descriptive analysis on the temporal development of topics can be obtained as a part of the website (see figure 2). Red line chart shows the total number of articles in a 4 hour time windows, the blue bar chart shows the number of news articles in 10 minute updates and the blue area shows the cumulative sum of articles in the story.

The website provides many more features for descriptive analysis of the articles. However, in order to extract more useful information from the system, indepth analysis with more sophisticated analytic methods is needed. The current paper focuses on two application areas, namely in the fields of polarity analysis and spatial-temporal analysis of news feeds.

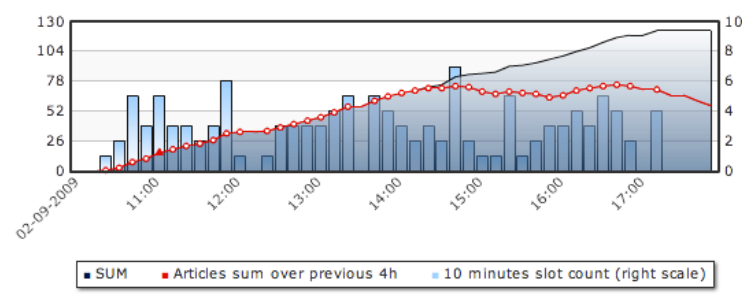

Figure 2: EMM Website Graph - Single story cluster evolution over time.

\section{POLARITY ANALYSIS}

In this section we are going to present a technique for polarity analysis on news streams. We demonstrate its usefulness on a sample of about 3 weeks of news data (approx. 15000 articles).

\subsection{Automatic Techniques}

To get a polarity score for each article, a basic analysis algorithm was applied. With the help of two lists with signal words (one containing words with a negative connotation and the other with positive ones) ${ }^{1}$ each word is classified as positive, negative or neu$\mathrm{tral}^{2}$. We count the number of positive signal words in an article and subtract the number of negative signal words from it. To improve the accuracy of the method, negation is taken into account. This is done by inverting the value of a word, if in a maximum distance of $X$ words a negation signal word is found (such as "no", "not", "without", ...). In this case, the parameter $X$ (the maximum distance to the negation signal word) was set to 3 , a value that experimentally proved as minimizing the failures.

Usually, the above mentioned technique is used in the context of sentiment analysis. Note that when we apply it to newspaper articles, we do not measure the author's opinion about the topic directly. However, what we measure is still related to sentiment in the broadest sense. Words with positive connotations arouse positive feelings about the topic and vice versa. To account for this difference in the semantics of the score, we call our analysis "polarity analysis" although classical sentiment analysis techniques are used.

In the last years, much research has been conducted in the area of sentiment analysis (see e.g., (Yu and Hatzivassiloglou, 2003; Wiegand and Klakow, 2008)). For document-level sentiment-analysis, which is what we do, classification algorithms are often used (such as Naive Bayes). In case of review analysis, many algorithms exist that additionally analyze the text with respect to what has been commented on (such as (Hu and Liu, 2004; Titov and McDonald, 2008)). So far, only few approaches exist that work on news articles, as this is a difficult domain. Please refer to (Pang and Lee, 2008) for a more comprehensive overview of sentiment and opinion analysis algorithms.

\footnotetext{
${ }^{1}$ The lists of signal words are taken from the General Inquirer Project: http://www.webuse.umd.edu:9090/

${ }^{2}$ Note that the list contains signal words of all parts of speech. That means, not only adjectives, but also nouns, verbs, etc. (e.g. "catastrophe", "to like").
} 
Using this simple algorithm has the advantage that the automatic analysis can be done fast. In our case, this is an important property, because we want to work on a streaming data set. However, note that the algorithm could easily be replaced, if the analysis task requires more sophisticated ones.

\subsection{Visual Representation of the Polarity Analysis results}

In analyzing the news feed, we are interested in the question how the different groups (e.g. countries) report on different topics. Do they share the same view on the topic with respect to the polarity of the articles? Are there clear differences between some countries? Does it depend on the topic how much they agree with each other? Which special observations can be made? What is challenging in this case, is that we cannot say clearly, what we are looking for. The fact that our dataset is not static, but that we are working with a data stream, aggravates the problem. Knowing what would be interesting to look at today, does not necessarily mean that this would also be a good view for tomorrow's news.

A good way to deal with this problem is to use an expressive visualization technique to represent the result of the automatic algorithm. Thanks to the great capabilities of the human visual system, large amounts of information can be grasped and processed at once if they are visualized. The automatic algorithms in the background make it possible that the tedious work of extracting the polarity of the text is left to the machine. The more demanding work of detecting patterns and anomalies in the data is done by the human analyst when interacting with the visualization.

Several visualization techniques for sentiment and opinion analysis exist. Among them is (Liu et al., 2005) that represents the result of attribute-based opinion analysis with bar charts. In (Gamon et al., 2005) reviews are clustered according to topic and the average opinion per cluster is visualized in a treemap representation. Morinaga et al. (Morinaga et al., 2002) use a 2D scatterplot to display the results of their automatic algorithm. A visualization technique that is able to show the temporal aspect of a data set is introduced in (Wanner et al., 2009). Note, that all approaches except for the last one are working on product reviews and not news.

We decided to apply the Literature Fingerprinting technique that was introduced in (Keim and Oelke, 2007). The advantage of this pixel-based visualization compared to the previously mentioned ones is that a large amount of values can be shown without the need of aggregation. Furthermore, the inherent hierarchy is clearly visible. In this technique, each score (here the polarity score) is represented by a single pixel and its value is mapped to the color of the pixel. Single pixels are grouped according to a given hierarchy (e.g. first according to topic and within the topics according to the location of the news agency).

\subsection{Visual Polarity Analysis}

Figure 3 shows a Literature Fingerprint for about three weeks (May 11th - May 28th 2009) of English news articles from all over the world. In the left column, each pixel represents the set of news articles for a single country. A block of pixels contains all the articles that belong to a specific topic. (Our topics are security, sports, and terrorism). Color is mapped to the average polarity score of the articles that are represented by the pixel (see color scale at the right). In the right column, the data are shown on a more detailed resolution level. In this case, each pixel represents a single article and the articles are first grouped according to the country they belong to, and then according to the topic they report on. Again, color is mapped to the polarity score, but this time it represents the score for a single article.

Looking at the left column of figure 3, it is easy to see that there are clear differences between the topics with respect to their fundamental tone. While security and terrorism show a negative trend for most countries, the opposite is the case for sports.

The advantage of the representation in the right column is that not only an average score is depicted. In the higher resolution level it can also be seen how many articles contributed to the average value and how homogeneous the reporting is with respect to the polarity that is expressed.

In the last line of the right column, three kinds of patterns can be perceived: 1) countries, for which almost all pixels are colored in shades of red (negative), 2) countries that are homogeneously shaded in blue or green, and 3) countries, in which all colors of our color scale occur. Among the ones whose articles were homogeneously classified as reporting negatively about terrorism are Australia, Croatia, and the Cayman Islands (see enlarged depiction at the bottom of the figure). A closer analysis shows that their articles are primarily about terrorism in other countries. Countries, in which a concrete danger of terroristic acts exists, usually show a multi-colored picture in our visualization with an overall negative tendency (see e.g. Great Britain or Israel). The reason for this is that political speeches or activities related to fight against terrorism are also included. This also nicely 


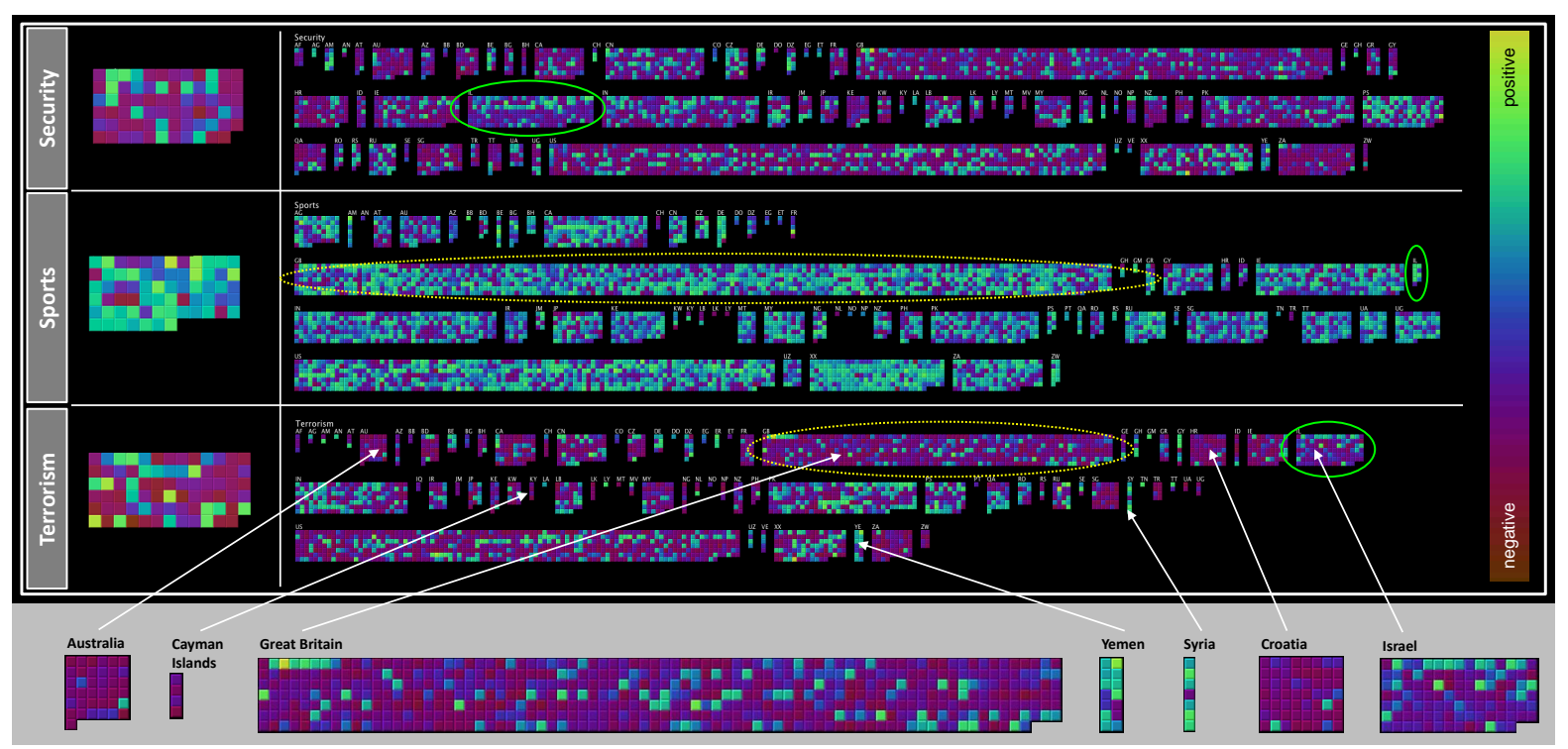

Figure 3: Polarity Analysis on news articles. In the right column, each pixel represents a single article. Its color is mapped to the polarity score. Articles published in the same country are grouped together into blocks of pixels. In the left column, each pixel represents the average polarity score for a set of articles of the same country. We display three different news categories.

exemplifies how our algorithm works. The latter articles are dominated by security-related terms, measures against terrorism, and optimistic perspectives for the future and thus our algorithm classifies them as positive, because the connotation of those terms is positive. This means that the used algorithm would not distinguish between an article that agrees with those political speeches and another one that cites them, but afterwards disassociates itself from the message. Finally, we were surprised to see that almost all articles in this category of the Syrian Arab Republic and Yemen are clearly classified as positive. Reading the articles revealed that in those days the foreign ministers of the Islamic countries met. Among other things, they discussed ways to preserve Islamic values and the Islamic culture, despite of experienced terrorist activities. For the participating countries, this was the major topic in those days and the optimistic tone of the conference (also praising their own countries' efforts and perspectives) explains the large amount of positive reports in the terrorism category.

Finally, we can also analyze the articles across topics. It is interesting to see that Great Britain has about twice as many articles in the category sports than in the category terrorism (see yellow dotted circles). Opposite to that, Israel has only very few articles in the sports category compared to the amount of articles in the categories terrorism and security (see green circles in figure 3 ).

\section{SPATIAL ANALYSIS}

The data provided by EMM can be regarded as an event-based multidimensional dataset, where each event represents one news item with a list of attributes. The first geographic attribute, the news origin, refers to the location of a news agency. Second, requiring more sophisticated tagging, is the location of the news' topics themselves. For the purpose of geographically tagging the location of a news item, the full text article is scanned for city, state and country names. When such a name is found in the document, its geographic location is automatically acquired from a look-up table. As a result, one new item could have more than one location, when more distinct places are mentioned.

\subsection{Application Challenges}

The most common approach to visually represent geographic information on a map is to pinpoint to its location. However, with large multidimensional data this task becomes a challenge. Data has to be shown using single pixels for each event and to map one attribute to the pixels' color (Keim et al., 2009). Such pixel-based visualization techniques are scalable but a well-known problem is that these techniques often have a high degree of overlap, which may occlude a significant portion of the data values shown. In addition, there might be a lack of correlation between information content and area size. 


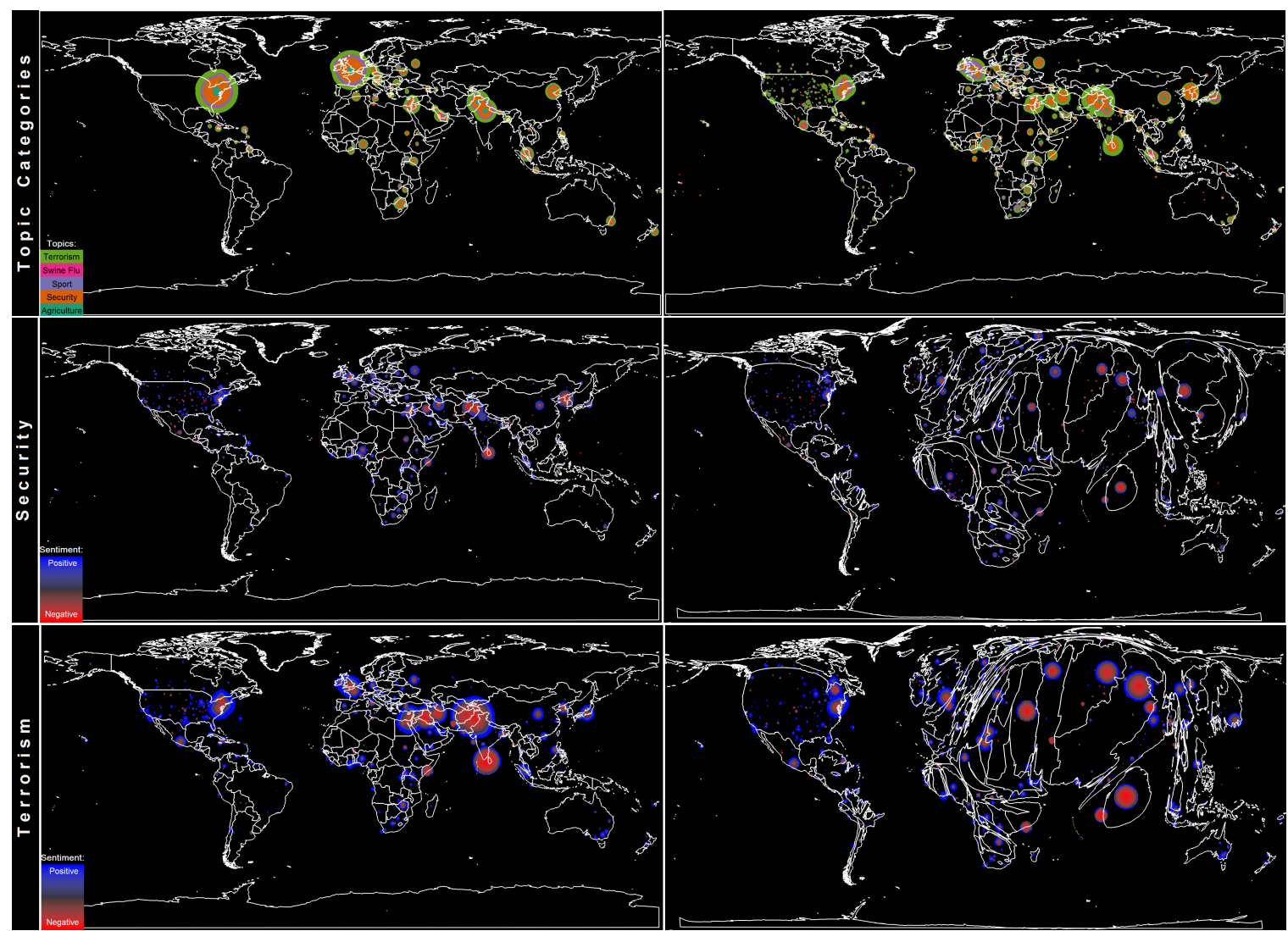

Figure 4: Spatial analysis of news feeds. (Top row) shows the spatial analysis of news feed for the news-origin (left) and news-location (right) for five selected categories. Topic categories are mapped to color. (Middle) and (Bottom rows) show the polarity of news on Security (middle row) and Terrorism (bottom row). Polarity levels are mapped to a bidirectional colormap having red for negative and blue for positive news. The Cartogram representations enhance regions of importance.

\subsection{Methods}

A number of different pixel-oriented visualization techniques have been proposed in recent years and shown to be useful for visually exploring data in many applications. These techniques differ mainly in their approach to arrange individual pixels in relation to each other, and in their choice of shaping the geographic regions to make maximal use of space.

In order to avoid overlapping pixels, we used in the current analysis a circular arrangement around the original location taking a given ordering of the elements into account (Bak et al., 2009a). The ordering usually corresponds to the coloring attribute starting with colors that occur least frequently. With this arrangement a natural looking visualization without artifacts is generated. The ordering of elements prevents randomly arranged points which would not benefit the user (Bak et al., 2009a).

Conventional data-plotting obscures data-points in densely populated areas, while sparsely populated areas waste space and hide the details of informa- tion. A way to obtain more space for regions with a high point density are Cartograms, which distort regions such that their size corresponds to a statistical attribute (Bak et al., 2009b; Tobler, 2004).

\subsection{Analysis Results}

The spatial aspect of the news was analyzed using the EMM data source with the techniques described previously. The data was obtained in the time period between May, 11th - June, 7th 2009. Figure 4 represents spatial analysis of the news feeds.

The top row shows the origin (left, where the news was published) and location (right, where the news took place) of news for five selected topics: agriculture, security, sport, swine flu and terrorism. The news originates mainly in Europe and in the US, and reported on the US, Europe, but also a lot on the Middle East and Asia. The topics of this time period were mainly dominated by Security and Terrorism.

The middle row shows the polarity score of news on security related topics. Polarity scores are mapped 
to color having red for negative, and blue for positive news with increasing intensity. The news mainly report on Central Asia and North Korea in the particular time period. The majority of these news is negative in their tonality. The Cartogram representation (right figure) enhances the area of these important locations.

The bottom row shows the polarity score of news on terrorism related topics. Polarity scores are mapped to color having red for negative, and blue for positive news with increasing intensity. The news mainly report on the Middle East and Central Asia, especially on the events in Sri-Lanka that occurred in the particular time period. Although the majority of the news is negative in its tonality, there are some positive reports on successes in the fight on terrorism. The Cartogram representation (right figure) enhances the area of these important locations.

\section{CONCLUSIONS}

The current paper describes an application framework for analyzing and exploring real-time news feed data. Polarity analysis showed how to assess the "tonality" of the published news articles using a technique called Literature Fingerprinting. The geospatial analysis demonstrated that many insights can be gained using pixel-based approaches. The great challenge for further research is to integrate respective techniques within the EMM-platform, make them scalable to large datasets, and to cope with real-time requirements.

\section{ACKNOWLEDGEMENTS}

This material is based upon work supported by the Science and Technology Directorate of the U.S. Department of Homeland Security under Grant Award Number 2008-ST-108-000002. The views and conclusions contained in this document are those of the authors and should not be interpreted as necessarily representing the official policies, either expressed or implied, of the U.S. Department of Homeland Security.

\section{REFERENCES}

Atkinson, M. and Van der Goot, E. (2009). Near real time information mining in mulitlingual news. In $W W W$ '09: Proc. 18th int. conference on World Wide Web, pages 1153-1154. ACM.
Bak, P., Keim, D. A., Schaefer, M., Stoffel, A., and Omer, I. (2009a). Spatiotemporal analysis of sensor logs using growth ring maps. In IEEE Trans. On Visualization And Computer Graphics. IEEE Press.

Bak, P., Mansmann, F., Janetzko, H., and Keim, D. A. (2009b). Density equalizing distrortion of large geographic point sets. In J. of Cartographic and Geographic Information Science, volume 36(3).

Gamon, M., Aue, A., Corston-Oliver, S., and Ringger, E. (2005). Pulse: Mining customer opinions from free text. In Advances in Intelligent Data Analysis VI, pages 121-132.

$\mathrm{Hu}, \mathrm{M}$. and Liu, B. (2004). Mining and summarizing customer reviews. In KDD '04: Proc. 10th ACM SIGKDD int. conference on Knowledge Discovery and Data Mining, pages 168-177. ACM.

Keim, D. A., Bak, P., and Schaefer, M. (2009). Dense pixel displays. In Encyclopedia of Database Systems. Springer.

Keim, D. A. and Oelke, D. (2007). Literature fingerprinting: A new method for visual literary analysis. In IEEE Symposium on Visual Analytics and Technology (VAST 2007), pages 115-122.

Liu, B., Hu, M., and Cheng, J. (2005). Opinion observer: analyzing and comparing opinions on the web. In WWW '05: Proc. 14th int. conference on World Wide Web, pages 342-351. ACM.

Morinaga, S., Yamanishi, K., Tateishi, K., and Fukushima, T. (2002). Mining product reputations on the web. In KDD '02: Proc. 8th ACM SIGKDD int. conference on Knowledge Discovery and Data Mining, pages 341349. ACM.

Pang, B. and Lee, L. (2008). Opinion mining and sentiment analysis. Foundations and Trends in Information Retrieval, 2(1-2):1-135.

Titov, I. and McDonald, R. (2008). A Joint Model of Text and Aspect Ratings for Sentiment Summarization. In Proceedings of ACL-08: HLT, pages 308-316. Assoc. for Computational Linguistics.

Tobler, W. R. (2004). Thirty five years of computer cartograms. In Association of American Geographers, volume 94(1), pages 58-73.

Wanner, F., Rohrdantz, C., Mansmann, F., Oelke, D., and Keim, D. A. (2009). Visual sentiment analysis of rss news feeds featuring the us presidential election in 2008. In Workshop on Visual Interfaces to the Social and the Semantic Web (VISSW 2009).

Wiegand, M. and Klakow, D. (2008). Optimizing language models for polarity classification. In Macdonald, C., Ounis, I., Plachouras, V., Ruthven, I., and White, R. W., editors, ECIR, volume 4956 of Lecture Notes in Computer Science, pages 612-616. Springer.

$\mathrm{Yu}, \mathrm{H}$. and Hatzivassiloglou, V. (2003). Towards answering opinion questions: separating facts from opinions and identifying the polarity of opinion sentences. In Proc. 2003 conference on Empirical methods in Natural Language Processing, pages 129-136. Assoc. for Computational Linguistics. 